\title{
New Type Ultra-Banach Spaces
}

\author{
Ibrahim Sanlibaba (Corresponding author) \\ Institute of Sciences, Nevsehir Haci Bektas Veli University \\ 50100, Nevşehir, Turkey \\ E-mail: ibrahimsanlibaba@gmail.com
}

\begin{abstract}
In this paper, we have given some definitions and theorems about ultra-metric, ultra-normed spaces which are shown in mathematical literature. Then, we have defined some new type ultra-Banach spaces and we have shown that these spaces are ultra-isomorphic and studied some interesting properties. Furthermore, some inclusion theorems are proved about these new type ultra-Banach spaces.
\end{abstract}

Keywords: Ultra-metric, ultra-norm, non-Archimedean space, ultra-convergent.

DOI: $10.7176 / \mathrm{JSTR} / 5-12-02$

\section{Introduction and Background}

One of the important branch as of the normed space theory is ultra-normed spaces. In general, we know that ordinary ultra-metric spaces are special kind of the metric spaces and one can obtain an ultra-normed space from the ultra-metric space. It can be seen that, in literature, the concept of ultra-metric is used in different areas of science, for instance, Flagg-Kopperman [1], Lemin [2], Priess-Crampe-Ribenboim [3] and Rammal [4]. Recently, Diagana has obtained some new type ultra-normed spaces and given some interesting properties of them [8-10].

In this paper, in the light of developments mentioned above, we have investigated a new type ultraBanach space. Now we will give some preliminary and definitions.

Let $X$ be a linear space, real valued function $\tilde{d}: X \times X X \mathbb{R}^{+}$and $\tilde{d}$ satisfies the following conditions:

$$
\begin{aligned}
& \left(d_{1}\right) \tilde{d}(x, y)=0 \text { if and only if } x=y, \\
& \left(d_{2}\right) \tilde{d}(x, y)=\tilde{d}(y, x), \\
& \left(d_{3}\right) \tilde{d}(x, y) \leq \max \{\tilde{d}(x, z), \tilde{d}(z, y)\}
\end{aligned}
$$

Then the function $\tilde{d}$ is called ultra-metric or super-metric on the set $X$. It is clear that, if the expression $\tilde{d}(x, y) \leq \max \{\tilde{d}(x, z), \tilde{d}(z, y)\}$ is held then $\tilde{d}(x, y) \leq \tilde{d}(x, z)+\tilde{d}(z, y)$ also held but vice versa is not true. The conditions $d_{1}-d_{3}$ are called ultra-metric conditions and the couple $(X, \tilde{d})$ is called ultra-metric or super-metric space. From here, we can say that every ultra-metric space is a metric space but vice versa is not true. Let $F$ be a field. Let us consider function $||:. F \rightarrow \mathbb{R}$ is satisfying following conditions:

$$
\begin{aligned}
& \left(a_{1}\right)|x| \geq 0 ;|x|=0 \text { if and only if } x=0 \\
& \left(a_{2}\right)|x y|=|x||y| \\
& \left(a_{3}\right)|x+y| \leq|x|+|y|
\end{aligned}
$$

then the function $|$.$| is called absolute value function and |x|$ is an absolute value of $x \in F$. In this case, the couple $(F,||$.$) is called Archimedean field [8].$ 
If we take

$$
\left(a_{3}\right)^{\prime}|x+y| \leq \max \{|x|,|y|\}
$$

instead of $\left(a_{3}\right)$ then the function |.| is called non-Archimedean absolute value. The condition $\left(a_{3}\right)^{\prime}$ is called strong triangle inequality or ultra-metric inequality. In this case, the field $F$ is also called as a nonArchimedean field. From here, the non-Archimedean absolute value we will be denoted with $\langle||$.$\rangle . A$ simple examples of Archimedean fields are $(\mathbb{R},||$.$) and (\mathbb{C},||$.$) , where the function |$.$| does not provide$ the condition $\left(a_{3}\right)^{\prime}$.

Example 1.1. Let $F$ be a field and consider the function

defined from $F \rightarrow \mathbb{R}^{+}$.

$$
\langle|x|\rangle= \begin{cases}1 & , x \neq 0 \\ 0 & , x=0\end{cases}
$$

In this case the function $\langle||$.$\rangle satisfies the conditions \left(a_{1}\right),\left(a_{2}\right)$ and $\left(a_{3}\right)^{\prime}$ so, the pair $(F,\langle||\rangle)$ is called trivial non-Archimedean field.

Example 1.2. Let us consider function $d: \mathbb{R x} \mathbb{R} \rightarrow \mathbb{R}^{+}$, defined as $d(x, y)=|x-y|$, where the notation |.| is absolute value function. Clearly, $d$ satisfies axioms of ordinary metric space. But the function $d$ does not satisfy the axiom $\left(a_{3}\right)^{\prime}$, therefore the couple $(\mathbb{R}, d)$ is not an ultra-metric space.

Let $X$ be a non empty set and consider function $\tilde{d}: X \mathrm{x} X \rightarrow \mathbb{R}^{+}$defined by

$$
\tilde{d}(x, y)=\left\{\begin{array}{l}
0, x=y \\
1, x \neq y
\end{array}\right.
$$

Then the function $\tilde{d}$ satisfies the conditions of ultra-metric, so $X$ is an ultra-metric space [9], [10]. Thus we can suggest that "Every discrete metric space is an ultra-metric space". Let $X$ be a linear space $F$ be a field and $x, y \in X, \alpha \in F$. If a single argument non negative real valued function $u$ satisfies the following conditions then $u$ is a called ultra-norm.

$\left(n_{1}^{\prime}\right) u(x)=0 \Leftrightarrow x=\theta$,

$\left(n_{2}^{\prime}\right) u(\alpha x)=|\alpha| u(x)$,

$\left(n_{3}^{\prime}\right) u(x+y) \leq \max \{u(x), u(y)\}$

It is clear that, if the inequality $u(x+y) \leq \max \{u(x), u(y)\}$ is held then the $u(x+y) \leq u(x)+u(y)$ is held but vice versa is not true. Let $u$ be an ultra-norm on $X$ and for all $x, y \in X$ we define function $\tilde{d}(x, y)=u(x-y)$ from $X \times X$ to $\mathbb{R}$. Then the function $\tilde{d}$ satisfies ultra-metric conditions [16]. This shows that every ultra-normed space are ultra-metric space.

Definition 1.1 Let us suppose that $(X, u)$ be an ultra-normed space and $\left(x_{k}\right)$ be a sequence $X$.

If for all $\varepsilon>0$ there exists a $k_{0}$ positive integer such that $u\left(x_{k}-x_{0}\right)<\varepsilon$ for all $k \geq k_{0}$, then $\left(x_{k}\right)$ is called ultra-convergent (or super-convergent) to $x_{0}$ and denoted by $\stackrel{u}{\rightarrow} x_{0}, k \rightarrow \infty$. If $x_{0}$ equal to zero then $\left(x_{k}\right)$ is called ultra-null(super-null) sequence.

(ii) The sequence $\left(x_{k}\right)$ is called ultra-Cauchy (or super-Cauchy), if for all $\varepsilon>0$ there exists a $k_{0}$ positive integer such that $u\left(x_{k}-x_{i}\right)<\varepsilon$ for all $k, i \geq k_{0}$.

(iii) The sequence $\left(x_{k}\right)$ is called ultra-bounded (or super-bounded), if $u\left(x_{k}\right) \leq K$ for $K \geq 0$.

(iv) Let $(X, u)$ be ultra-normed space. If every ultra-Cauchy sequence in $(X, u)$ ultra-convergent to $x_{0} \in X$ then $X$ called ultracomplete(or ultraBanach) space, [17].

Let $F$ be a non-Archimedian field. In this case set $w=\left\{\left(x_{k}\right): f: I N \rightarrow F, f(k)=x_{k}\right\}$ is called the sets 
of all sequences. The sets of ultra-bounded, ultra-convergent and ultra-null sequences are denoted by $l_{\infty}(F), c(F)$ and $c_{0}(F)$, respectively and defined as follow,

$$
\begin{aligned}
& l_{\infty}(F)=\left\{x=\left(x_{k}\right) \in w: \sup _{k \in \mathbb{N}}\left\langle\left|x_{k}\right|\right\rangle<\infty\right\}, \\
& c(F)=\left\{x=\left(x_{k}\right) \in w: x_{k} \stackrel{u}{\rightarrow} x_{0}, k \rightarrow \infty, x_{0} \in F\right\}, \\
& c_{0}(F)=\left\{x=\left(x_{k}\right) \in w: x_{k} \stackrel{u}{\rightarrow} 0 k \rightarrow \infty\right\} .
\end{aligned}
$$

This shows that the spaces $l_{\infty}(F), c(F)$ and $c_{0}(F)$ are ultra-Banach spaces with the norm defined by

$$
u_{l_{\infty}(F)}(x)=u_{c(F)}(x)=u_{c_{0}(F)}(x)=\sup _{k}\left\langle\left|x_{k}\right|\right\rangle
$$

where $|$.$| is satisfies the property \left(a_{3}\right)^{\prime}$. Some new type ultra-normed sequence spaces defined by Diagana [8-10] as follows:

$$
\begin{aligned}
& l_{\infty}(F, \rho)=\left\{x=\left(x_{k}\right) \in w: \sup _{k \in \mathbb{N}}\left\langle\left|x_{k}\right|\right\rangle \rho_{k}<\infty\right\}, \\
& c(F, \rho)=\left\{x=\left(x_{k}\right) \in w: \lim _{k}\left\langle\left|x_{k}\right|\right\rangle \rho_{k} \text { exists }\right\}, \\
& c_{0}(F, \rho)=\left\{x=\left(x_{k}\right) \in w: \lim _{k}\left\langle\left|x_{k}\right|\right\rangle \rho_{k}=0\right\}
\end{aligned}
$$

and these are ultra-Banachspaces with defined ultra-norm

$$
u_{l_{\infty}(F, \rho)}(x)=u_{c(F, \rho)}(x)=u_{c_{0}(F, \rho)}(x)=\sup _{k}\left\langle\left|x_{k}\right|\right\rangle \rho_{k} .
$$

since every ultra-normed space is a normed space, if take $\rho_{k}=1$ for all $k$, then the spaces $l_{\infty}(F, \rho)$, $c(F, \rho)$ and $c_{0}(F, \rho)$ are reduced to, $l_{\infty}(F), c(F)$ and $c_{0}(F)$ respectively. In other words, the spaces $l_{\infty}(F, \rho), c(F, \rho)$ and $c_{0}(F, \rho)$ are large than the spaces $l_{\infty}(F), c(F), c_{0}(F), l_{\infty}, c$ and $c_{0}$, where $l_{\infty}, c$ and $c_{0}$ denotes ordinary bounded, convergent and null sequence spaces in Achimedian field, respectively. Now, we will give a new definition.

Definition 1.2 (Ultra-isometry) Let $X$ and $Y$ be vector space on non Archimedian field $F$ and the mapping $T: X \rightarrow Y$ be given. If, for all $x \in X, u(T x)_{Y}=u(x)_{X}$ then the map $T$ is called ultra-isometry from $X$ to $Y$. In this case, the spaces $X$ and $Y$ are called as ultra-isometric spaces. In addition, if the mapping $T$ is bijective, then the spaces $X$ and $Y$ are called as ultra-isomorphic spaces and denoted by $X \cong Y$ [17].

\section{Zweier Type Ultra-normed Spaces}

Each linear subspace of $w$ is called a sequence space. Let $\lambda$ and $\mu$ be two sequence spaces and $A=$ $\left(a_{n k}\right)(\mathrm{n}, \mathrm{k}=0,1,2, \ldots)$ be an infinite matrix of real or complex numbers $a_{n k}$. Then, we can say that $A$ defines a matrix mapping from $\lambda$ to $\mu$, and we denote it by writing $A: \lambda \rightarrow \mu$ if for every sequence $\left(x_{k}\right) \in$ $\lambda$ the sequence $A x=\left\{(A x)_{n}\right\}$ the $A$ - transform of $x$ is in $\mu$ where

$$
(A x)_{n}=\sum_{k} a_{n k} x_{k}
$$

By $(\lambda: \mu)$, we denote the class of matrices $A$ such that $A: \lambda \rightarrow \mu$ Thus, $A \in(\lambda: \mu)$, if and only if the series on the right side of (2.1) converges for each positive integer $n$ and every $\left(x_{k}\right) \in \lambda$ we have $A x=$ $\left\{(A x)_{n}\right\} \in \mu$ for all $\left(x_{k}\right) \in \lambda$.

The matrix domain $\lambda_{A}$ of an infinite matrix $A$ in a sequence space $\lambda$ is defined by

$$
\lambda_{A}=\left\{\mathrm{x}=\left(\mathrm{x}_{\mathrm{k}}\right) \in \mathrm{w}: \mathrm{Ax} \in \lambda\right\}
$$

If we take $\lambda=c$ then $c_{A}$ is called convergence domain of $A$.

By using of the matrix domain of a particular limitation method so many sequences spaces have been 
built and published in many maths journals. By reviewing the literature, one can reach them easily ( for instance, see Altay and Başar [11-13], Kirişçi and Başar [14], Şengönül and Kayaduman [5], Şengönül [6]. Finally, the new technique for deducing certain topological properties, such as AB-, KB-, ADproperties, solidity and monotonicity etc., and determining the $\alpha-, \beta-\$$ and $\gamma-$ duals of the domain of a triangle matrix in a sequence space is given by Altay and Başar [11], [12]. [13].

Definition 2.1. Let $n, k \in \mathbb{N}$ and consider infinite matrix $Z=\left(z_{n k}\right)$ defined by

$$
z_{n k}=\left\{\begin{array}{rr}
p, & n=k \\
1-p, & n-1=k, p \in \mathbb{R}-\{-1\} . \\
0, & \text { others }
\end{array}\right.
$$

The infinite matrix $Z=\left(z_{n k}\right)$ is called Zweier matrix, [9]. Let $x=\left(x_{k}\right)$ be a sequence and $Z$-transform of $x=\left(x_{k}\right)$ is defined as follows [6]:

$$
y_{k}=(Z x)_{k}=p x_{k}+(1-p) x_{k-1}
$$

Now we will define some new type ultra-normed sequence spaces as follows:

$$
\begin{aligned}
& \tilde{c}_{0}(Z, F)=\left\{x: p x_{k}+(1-p) x_{k-1} \stackrel{u}{\rightarrow} x_{0}, k \rightarrow \infty\right\}, \\
& \tilde{c}(Z, F)=\left\{x: p x_{k}+(1-p) x_{k-1} \stackrel{u}{\rightarrow} x_{0}, k \rightarrow \infty, x_{0} \in F\right\}, \\
& \tilde{l}_{\infty}(Z, F)=\left\{x: \sup _{k}\left\langle\left|p x_{k}+(1-p) x_{k-1}\right|\right\rangle<\infty\right\} .
\end{aligned}
$$

It is clear that the sets $\widetilde{c_{0}}(Z, F), \widetilde{c}(Z, F)$ and $\tilde{l}_{\infty}(Z, F)$ are defined with $Z x$ transforms in $c_{0}(F), c(F)$ and $l_{\infty}(F)$, respectively. And these spaces are ultra (super) normed spaces defined by ultra (super) norm;

$$
u_{\tilde{l}_{\infty}(Z, F)}(x)=\sup _{k}\left\langle\left|p x_{k}+(1-p) x_{k-1}\right|\right\rangle
$$

If we write $\tilde{\lambda}(Z, F) \in\left\{\widetilde{c_{0}}(Z, F), \widetilde{c}(Z, F), \tilde{l}_{\infty}(Z, F)\right\}$ and $\lambda(F) \in\left\{c_{0}(F), c(F), l_{\infty}(F)\right\}$ then we can give a theorem as follows:

Theorem 2.2. Let us consider transformation

$$
\begin{aligned}
& \mathrm{Z}: \tilde{\lambda}(Z, F) \rightarrow \lambda(F), \\
& \quad x \rightarrow \mathrm{Zx}=\mathrm{y}, \quad \mathrm{y}=\mathrm{y}_{k}, \mathrm{y}_{k}=p x_{k}+(1-p) x_{k-1} .
\end{aligned}
$$

In this case, the map $Z$ is ultra-isomorphism and the spaces $\tilde{\lambda}(Z, F)$ and $\lambda(F)$ are also ultra-isometric.

Proof. Let us suppose that $x, y \in \tilde{\lambda}(Z, F)$ and $\alpha \in F$. It is clear that $Z$ is linear. Furthermore, if $\mathrm{Zx}=\theta$, so $p x_{k}+(1-p) x_{k-1}=\theta$ then $Z^{-1}(\mathrm{Zx})=Z^{-1} \theta$ and we obtain that $x=\theta$. This result shows to us the transformation $\mathrm{Z}$ is one to one from $\tilde{\lambda}(F)$ to $\lambda(F)$. Since every element of the space $\tilde{\lambda}(F)$ is obtainied from transform of $x \in \tilde{\lambda}(F)$, it is clear that $\mathrm{Z}$ is onto. Secondly, we must show that the transform $\mathrm{Z}$ preserves ultra-norm between the spaces $\tilde{\lambda}(F)$ and $\lambda(F)$. For this, let us define the sequence $x_{k}=$ $\sum_{j=0}^{k}(-1)^{k-j} \frac{(1-p)^{k-j}}{p^{k-j+1}} y_{j},[15,17]$

$$
\begin{aligned}
u(x)= & \sup _{k}\left\langle\left|p x_{k}+(1-p) x_{k-1}\right|\right\rangle \\
= & \sup _{k}\left\langle\left|p \sum_{j=0}^{k}(-1)^{k-j} \frac{(1-p)^{k-j}}{p^{k-j+1}} y_{j}+(1-p) \sum_{j=0}^{k}(-1)^{k-j} \frac{(1-p)^{k-j}}{p^{k-j+1}} y_{j}\right|\right\rangle \\
& =\sup _{k}\left\langle\left|y_{k}\right|\right\rangle=\sup _{k}\langle|Z x|\rangle=u(\mathrm{Z} x) .
\end{aligned}
$$

Then here the proof ends. 
Theorem 2.3. The spaces $\widetilde{c_{0}}(Z, F), \tilde{c}(Z, F), \tilde{l}_{\infty}(Z, F)$ are ultra-Banach spaces defined by ultra-norm (2.4).

Proof. Since proofs are similar, we have only considered $\tilde{l}_{\infty}(Z, F)$. It is clear that ultra-norm defined by (2.4) satisfies the conditions $\left(n_{1}^{\prime}\right),\left(n_{2}^{\prime}\right)$ and $\left(n_{3}^{\prime}\right)$.

Let suppose that $\left(x^{n}\right)$ be ultra-Cauchy in $\tilde{l}_{\infty}(Z, F)$. In this case, we see that there exists a positive integer $n_{0}$ such that

$$
\begin{aligned}
u_{\tilde{l}_{\infty}(Z, F)}\left(x_{k}{ }^{m}-x_{k}{ }^{n}\right)= & \sup _{k}\left\langle\left|\left(p x_{k}{ }_{k}+(1-p) x_{k-1}^{m}\right)-\left(p x_{k}^{n}+(1-p) x_{k-1}^{n}\right)\right|\right\rangle \\
& =u_{l_{\infty}(Z, F, \rho)}\left(Z\left(x_{k}{ }^{m}-x_{k}{ }^{n}\right)\right)<\varepsilon \quad \text { for } \quad m, n \geq n_{0} .
\end{aligned}
$$

From her we can write

$$
\left\langle\left|\left(p x_{k}^{m}+(1-p) x^{m}{ }_{k-1}\right)-\left(p x_{k}^{n}+(1-p) x_{k-1}^{n}\right)\right|\right\rangle=\left\langle\left|\left(Z\left(x_{k}{ }^{m}-x_{k}{ }^{n}\right)\right)\right|\right\rangle\langle\varepsilon .
$$

This shows to us, the sequence $\left(Z\left(x_{k}{ }^{m}-x_{k}{ }^{n}\right)\right)$ is a ultra-Cauchy sequence in $l_{\infty}(F)$. If we take into account $l_{\infty}(F)$ is ultra-complete and the space $l_{\infty}(F)$ and $\tilde{l}_{\infty}(Z, F)$ are linearly ultra-isometric, then we can easily claim that every ultra-Cauchy sequence in $\tilde{l}_{\infty}(Z, F)$ is convergent. Let us suppose that $x_{k}^{n}$ $\stackrel{u}{\rightarrow} x_{k}, n \rightarrow \infty, k=0,1,2, \ldots$. Now we will show that the sequence $x=\left(x_{k}\right) \in \tilde{l}_{\infty}(Z, F)$. We know that from Theorem 2.1, the mapping $Z$ is bijective from $\tilde{l}_{\infty}(Z, F)$ to $l_{\infty}(F)$. It is deduce that $x=\left(x_{k}\right) \in$ $\tilde{l}_{\infty}(Z, F)$.

Consequently the space $\left(\tilde{l}_{\infty}(Z, F), u\right)$ is a ultra-Banach space.

Theorem 2.4. The inclusions

$$
\begin{aligned}
& \left(i_{1}\right) \tilde{l}_{\infty}(Z, F) \subseteq l_{\infty}(F) \\
& \left(i_{2}\right) \widetilde{c_{0}}(Z, F) \subseteq \tilde{c}(Z, F) \subseteq \tilde{l}_{\infty}(Z, F) \\
& \left(i_{3}\right) c_{0} \subseteq \widetilde{c_{0}}(Z, F) \text { and } c \subseteq \tilde{c}(Z, F) \text { are hold. }
\end{aligned}
$$

Proof. The proof of the $\left(i_{2}\right)$ and $\left(i_{3}\right)$ are clear so we will give a proof for only $\left(i_{1}\right)$.

$\left(i_{1}\right)$ If $x \in \tilde{l}_{\infty}(Z, F)$, then

$$
\begin{aligned}
u(x)_{\tilde{l}_{\infty}(Z, F)}= & \sup _{k}\left\langle\left|p x_{k}+(1-p) x_{k-1}\right|\right\rangle \leq \sup _{k}\left\{\max \left\{\left|p x_{k}\right|,\left|(1-p) x_{k-1}\right|\right\}\right\} \\
& =K \sup _{k}\left\{\max \left\{\left|x_{k}\right|,\left|x_{k-1}\right|\right\}\right\}=K u_{l_{\infty}(Z, F)}(x)
\end{aligned}
$$

where $K=\max \{|p|,|1-p|\}$. This shows to us $x \in l_{\infty}(F)$. Similarly, we can easily prove that the inclusions $\tilde{c}(Z, F) \subseteq c(F)$ and $\widetilde{c_{0}}(Z, F) \subseteq c_{0}(F)$ are hold.

\section{Open Problems}

1- Lets us suppose that $A=\left(a_{n k}\right)$ be an infinite matrix and $\mathrm{x}=\left(x_{k}\right)$ be sequence in $l_{\infty}(F)$ (or $\left.c(F), c_{0}(F)\right)$. Then when does $(A x) \in l_{\infty}(F)$ for all $\mathrm{x}=\left(x_{k}\right) \in l_{\infty}(F)$ ? And other classes?

2-Similarly to the open problem 1 , when does $(A x) \in \tilde{l}_{\infty}(Z, F)$ for all $x=\left(x_{k}\right) \in \tilde{l}_{\infty}(Z, F)$ ?

3- If $\lambda$ is super-Banach space then the sets

$$
\begin{aligned}
& \lambda^{\alpha}=\left\{a=\left(a_{k}\right): a x \in c s(F) \text { for all } x \in \lambda\right\} \\
& \lambda^{\beta}=\left\{a=\left(a_{k}\right): a x \in b s(F) \text { for all } x \in \lambda\right\} \\
& \lambda^{\gamma}=\left\{a=\left(a_{k}\right): a x \in l(F) \text { for all } x \in \lambda\right\}
\end{aligned}
$$

are called $\alpha-, \beta-$, and $\gamma$-duals of the super-Banach space $\lambda$. 
In this case, what is the $\alpha-, \beta-$, and $\gamma-$ duals of the spaces $l_{\infty}(F), c(F), c_{0}(F)$ other spaces?

\section{Conclusions}

In this paper, we have obtained a new type ultra-Banach space is called Zweier ultra-Banach space and investigated some inclusisons theorems.

\section{Acknowledgment}

Thanks to Sevda ATPINAR, from Nevşehir Hacı Bektaş Veli University- Department of mathematics, for correcting some grammatical errors in the manuscript.

\section{References}

Flagg, B. and Kopperman R. (1997). Computational models for ultrametric spaces, in "XIII Conference on Mathematical Foundations of Programming Semantics", , pp. 83-92,

Lemin, V. A. (2001). Finite ultrametric spaces and computer science, in "Categorical Perspectives", ed. A. Melton and U.Koslowski (Trends in Mathematics, vol. 16) BirkhauserVerlag, Boston Basel - Berlin, , pp. 219-243.

Priess, S. -Crampe P. Ribenboim.(2000). Ultrametric spaces and logic programming, J. of Logic Programming 42:2, 59-70.

Rammal, R. and Toulouse, G., M. A. (1986).Virasoro, Ultrametricity for physicists, Rev. Modern

Physics 58 765-788.

Şengönül, M. and Kayaduman, K. (2012). On the Riesz almost convergent sequences spaces, Abstract and Applied Analysis, 691694.

Şengönül, M. (2007). On The Zweier Sequence Space. Demontratio Mathematica. Vol:XL, No:1.

Altay, B. and Başar, F. (2007). Certaintopological properties and duals of the matrix domain of a triangle matrix in a sequence space, J. Math. Anal. Appl. 336(1) 632645.

Diagana, T., (2006). $\mathrm{c}_{0}$-Semigroups of Linear Operators on some Ultrametric Banach Space, Hindawi Publishing Corporation International Journal of Mathematics and Mathematical Sciences Volume, DOI10. 1155/2006/52398,

Diagana, T. (2007). Non-Arcimedean Linear Operators and Applications, by Nova Science Publishers, Inc.

Diagana, T. (2006). An Introduction to Classical and p-ADIC Theory of Linear Operators and Applications, by Nova Science Publishers, Inc.

Altay, B. and Başar, F. (2005). Some Euler sequence spaces of non-absolutetype, Ukrainian Math. J. 57 (1) $1-17$.

Altay, B. and Başar, F. (2006). Some paranormed Riezs sequence spaces of non-absolute type, Southeast Asian Bull. Math. 30(5) 591608.

Altay, B. and Başar, F. (2006).Some paranormed sequence spaces of non-absolute type derived by weighted mean, J. Math. Anal. Appl.319(2), 494-508.

Kirişçi, M. and Başar, F. (2010). Some new sequence spaces derived by the domain of generalized difference matrix , Comput. Math. Appl. 60 (5) 1299—1309, 
Schikhof, W. H. (2002). Ultrametric Functional Analysis Seventh International Conference on p-o6lc Functional Analysis June 17-21, University of Nijmegen, The Netherlands.

Boos, J. (2000). Classical and Modern Methods in Summability, Oxford University Press Inc., New York.

Şanlıbaba, I. (2014). Ultrametricbanach space isomorphic to new spaces. Master's thesis, Nevşehir Hacı Bektaş Veli University, Nevşehir. 